\title{
Réalisation d'un diagnostic des ouvrages de défense contre la mer du littoral Vendéen
}

\author{
Jacques Piallat $^{1}$, Didier Labey ${ }^{2}$ \\ ${ }^{I}$ Directeur de projet, Service Hydraulique Fluviale et Maritime \\ BCEOMEAU - 78, allée John Napier - 34965 Montpellier Cedex2 \\ Téléphone: 0467992200 e-mail: j.piallat@bceom.fr \\ ${ }^{2}$ Directeur de projet, ANTEA - Agence Bretagne-Pays-de-la Loire \\ 8, boulevard Albert Einstein - BP 32345 - 44323 Nantes Cedex3 \\ Téléphone:0228013232 e-mail:d.labey@antea.brgm.fr
}

\section{Résumé}

Les tempêtes de fin 1999 ont révélé la fragilité des ouvrages de défense contre la mer. En particulier, la sécurité des habitants pourrait être affectée dans les secteurs poldérisés que l'on trouve en baie de Bourgneuf, à Noirmoutier et dans l'anse de l'Aiguillon. L'étude, basée sur un inventaire des ouvrages et des reconnaissances de sol, a permis d'établir un classement typologique puis le diagnostic des ouvrages (150 km de littoral). L'analyse des niveaux d'eau extrêmes a montré que certaines zones étaient exposées à des surcotes importantes. Cette étude a conclu sur des principes de restauration et de remise à niveau des ouvrages, avec définition des priorités pour les travaux urgents (10 millions d'Euros). Une méthode de maintien de connaissance du patrimoine littoral a été élaborée.

\begin{abstract}
$\underline{\text { Abstract }}$
The storms of the year 1999 have stressed the fragility of the coastal protection devices. In particular, it seems that the population safety could be affected in the polder areas of the Bourgneuf bay, in Noirmoutier and in the Aiguillon bight as well. The present study, based on an inventory of the engineering works and on soil [samples], allowed to define a typological classification, then the works diagnosis on a $150 \mathrm{~km}$ coastline length. The analysis of the highest water levels showed that some of the areas are subjected to significant surges. The study conclusions lead to restoration and upgrading principles for the works, along with the definition of priorities for the urgent works to be carried out (10 M Euros). A method for keeping the knowledge of the coastal heritage has been issued.
\end{abstract}

\section{Déroulement de l'étude}

La zone d'étude couvre $150 \mathrm{~km}$ de côtes du département de la Vendée et comprend 4 secteurs géographiques: le littoral continental de la baie de Bourgneuf (25 km); l'île de Noirmoutier dans son ensemble $(56 \mathrm{~km})$; l'anse de l'Aiguillon (34 $\mathrm{km})$; la Vendée continentale entre Saint-Jean-de-Monts et Brem-sur-Mer $(34 \mathrm{~km})$. 
Les ports conchylicoles du Bec et du Brochet sont traités à part vu les urgences. L'étude a été réalisée, secteur par secteur, en 4 phases:

$\checkmark$ Inventaire des ouvrages et classement typologique - exposition aux actions hydrodynamiques.

$\checkmark$ Etude de diagnostic des ouvrages.

$\checkmark$ Principe de restauration et schéma de définition des urgences.

$\checkmark$ Méthodologie de maintien de la connaissance du patrimoine littoral

L'analyse des niveaux extrêmes et de la surélévation contemporaine du niveau de la mer a été traitée à part.

\section{La surélévation du niveau de la mer - Niveau extrême de référence}

Le réchauffement de l'atmosphère entraîne un certain nombre de bouleversements climatiques, et entre autres conséquences, une surélévation du niveau de la mer. Quelle répercussion en attendre sur le domaine littoral et les ouvrages maritimes ? Comment la prendre en compte dans le diagnostic des ouvrages et les propositions de restauration ? Ces questions sont d'autant plus importantes que les niveaux maxima de la mer observés ces dernières années dépassent les valeurs jusqu'à présent admises [1]. Cependant, comme les surcotes les plus élevées ont rarement coïncidé avec des pleines mers de vives-eaux [2], il est donc statistiquement probable que des niveaux d'eaux extrêmes plus élevés que ceux observés récemment se produiront dans un avenir proche. Le parti pris dans cette étude a été de prendre comme niveau d'eau de projet, le niveau d'eau "historique" issu des observations, augmenté de l'élévation attendue du niveau moyen des océans, et d'une marge plus ou moins importante pour intégrer des effets de site.

Compte tenu des évolutions récentes constatées à Brest (mesures des niveaux moyens) [3], et des résultats des simulations numériques menées par l'IPCC [4], on peut conclure que l'augmentation moyenne du niveau de la mer sera de l'ordre de 2 $\mathrm{mm}$ par an pour la décennie en cours, avec une probable accélération dans les prochaines décennies. Les valeurs retenues dans cette étude sont les suivantes :

$\checkmark$ élévation de $20 \mathrm{~cm}$ pour 2050 ,

$\checkmark$ élévation de $50 \mathrm{~cm}$ d'ici la fin du XXIème siècle.

3. Inventaire des ouvrages et classement typologique, exposition aux actions hydrodynamiques

\subsection{Exposition aux actions hydrodynamiques}

Les zones d'études ont été découpées en secteurs homogènes vis à vis des niveaux d'eau extrêmes et des expositions aux agitations. Les courants n'ont pas été pris en compte dans ce découpage car peu significatifs après analyse des sites. Pour chaque zone homogène, une fiche type a été établie (figure 1). Elle donne les niveaux d'eau (marées astronomiques et niveaux d'eau extrêmes de projet), l'exposition aux houles et mers du vent, et une analyse de l'altimétrie des ouvrages vis à vis des conditions hydrodynamiques (franchissements - surverses). 


\section{VII ${ }^{e ̀ m e s}$ Journées Nationales Génie Civil - Génie Côtier, Anglet, France, 15-17 Mai 2002}

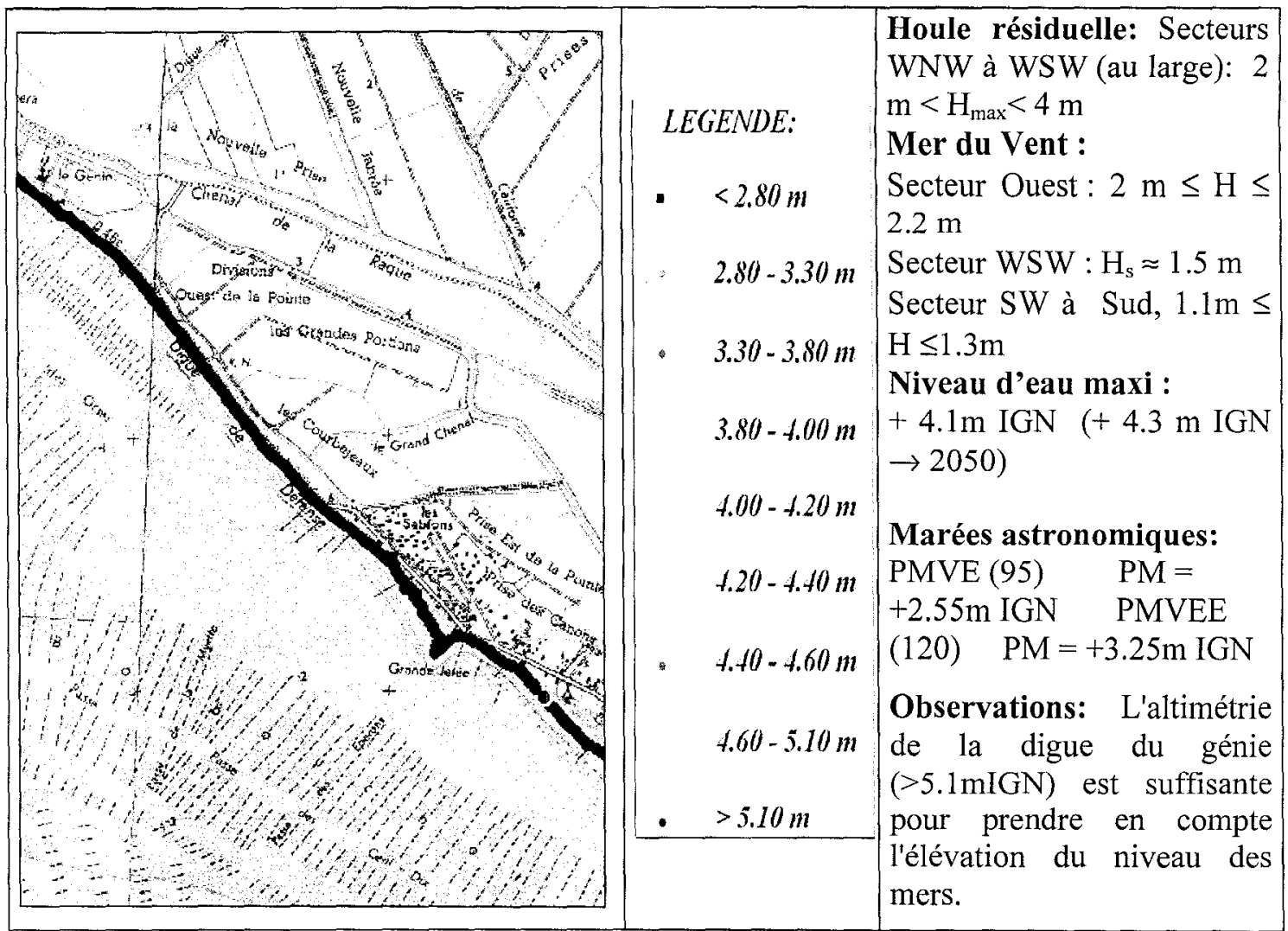

Figure 1. Exemple de fiche altimétrie: expositions aux actions hydrodynamiques -digue du Génie, anse de l'Aiguillon-

Les valeurs statistiques des niveaux extrêmes pour une période de retour de 100 ans [4] donnent $+3.8 \mathrm{~m}$ IGN en baie de Bourgneuf et $+3.9 \mathrm{~m}$ IGN dans l'anse de l'Aiguillon. Ces valeurs sont à rapprocher du niveau de pleine mer (PM) de coefficient 120 qui est $+3.3 \mathrm{~m}$ IGN pour ces deux zones. Un niveau d'eau centennal se situe donc $1 / 2 \mathrm{~m}$ au-dessus du niveau de PM de coefficient 120 . Les surcotes associées sont de $1 \mathrm{~m}[1]$.

Les observations des niveaux d'eau lors des tempêtes jumelles du 26 et 27 décembre 1999 sont supérieures à $+4.1 \mathrm{~m}$ IGN. La surcote a été évaluée à $1.4 \mathrm{~m}$ en baie de Bourgneuf le 26-12-99, et à $2 \mathrm{~m}$ à l'embouchure de la Sèvre Niortaise le 27 12-99. Ces valeurs, supérieures aux valeurs admises pour la période de retour de 100 ans [1] [4], sont survenues lors d'une phase de déchet de la marée (respectivement coefficient 94 et 79). Ces surcotes sont à relier à des conditions météorologiques extrêmes (vents de mer $>150 \mathrm{~km} / \mathrm{h}$ ) qui ont provoqué une "onde de tempête", comparable à celle générée dans un cyclone. Les effets de site ont été exacerbés par la morphologie du littoral (les deux baies sont ouvertes face à l'Ouest), et par les vents qui étaient orientés suivant l'axe le plus défavorable.

L'exposition aux houles atlantiques et aux mers du vent dépend de la situation de l'ouvrage (sur la côte atlantique: houle et mer du vent; en baie: mer du vent) et de la hauteur d'eau en pied d'ouvrage. L'inventaire a montré que la totalité des "ouvrages" découvre largement à partir de la mi-marée, certains étant sur le 
haut estran et seulement atteints par les jets de rive lors des tempêtes (perrés bas, ouvrages "naturels" comme les dunes). Pour les sites exposés aux houles atlantiques, la hauteur des vagues est limitée par déferlement en pied d'ouvrage $(\mathrm{H}=0.78 \mathrm{~d})$, et à des valeurs faibles, pour les mers du vent dans la baie de Bourgneuf et l'anse de l'Aiguillon $(\mathrm{H} \leq 2 \mathrm{~m})$.

Le niveau maxima atteint par la mer est un facteur déterminant, que se soit pour la pérennité des ouvrages (actions des vagues) ou vis-à-vis des franchissements et débordements.

\subsection{Inventaire des ouvrages}

L'ensemble du linéaire de rivage a été parcouru à pied par une équipe de 2 à 3 personnes. ANTEA s'est occupé plus particulièrement de l'environnement géotechnique, des fondations et de la structure interne de l'ouvrage et le BCEOM de sa tenue vis-à-vis des contraintes hydrodynamiques. L'étude sur le terrain a été complété par des reconnaissances topographiques pour lever les profils en travers des ouvrages et compléter les données sur l'altimétrie de la crête de l'ouvrage. Des reconnaissances de sol ont eu pour objectif une meilleure connaissance de la nature du noyau des ouvrages (ouvrage en terre) et du terrain sous-jacent. Les données existantes sur les ouvrages ont été recueillies auprès des divers services (DDE, CG85, Syndicats).

Chaque ouvrage a été décrit par une fiche typologique (figure 2)donnant sa situation, montrant une coupe type (levé topographique) ou un schéma type (figure 3 ), et complétée par des photos prises suivant des angles déterminés à l'avance.

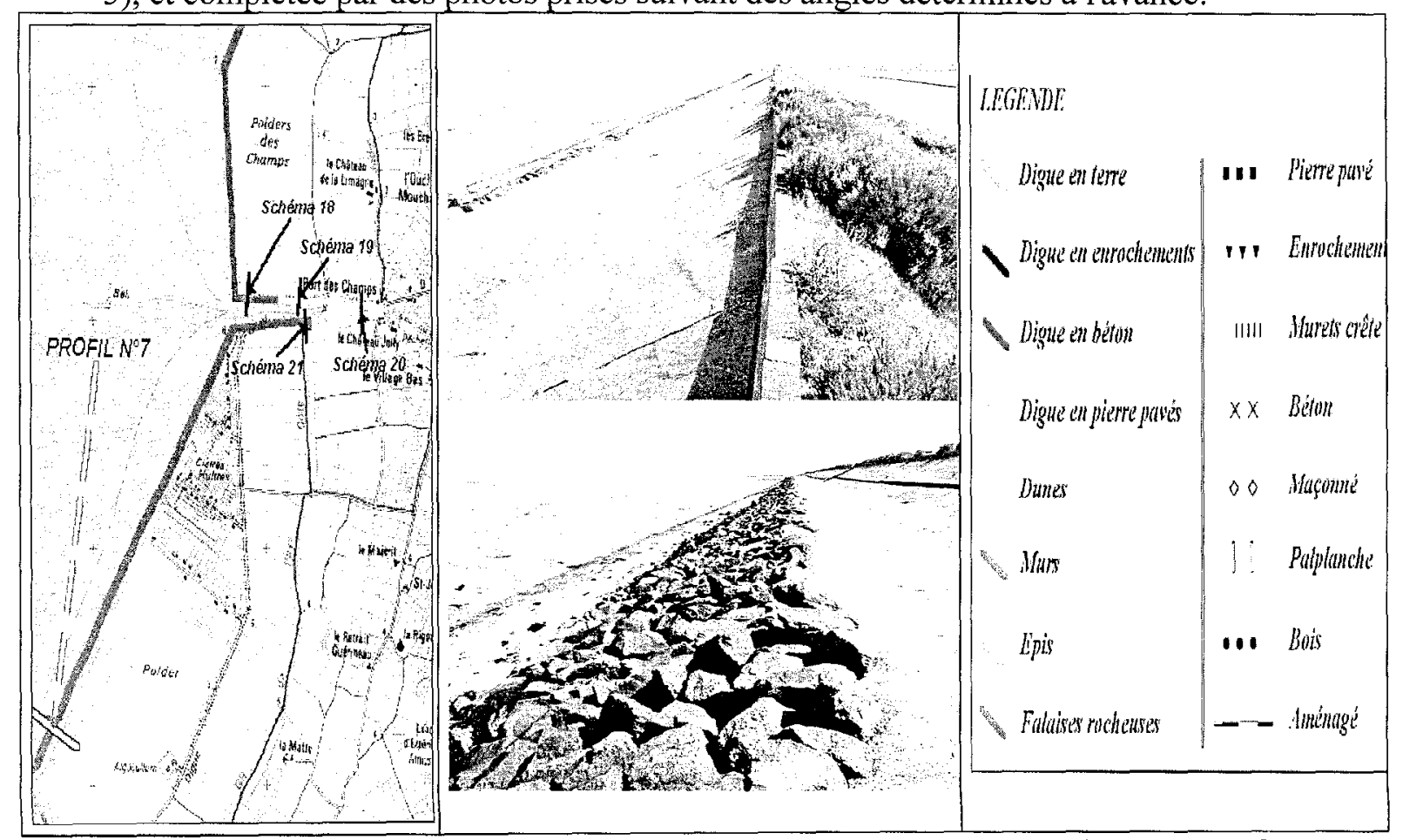

Figure 2. Fiche typologique - Digue du polder des Champs - baie de Bourgneuf 


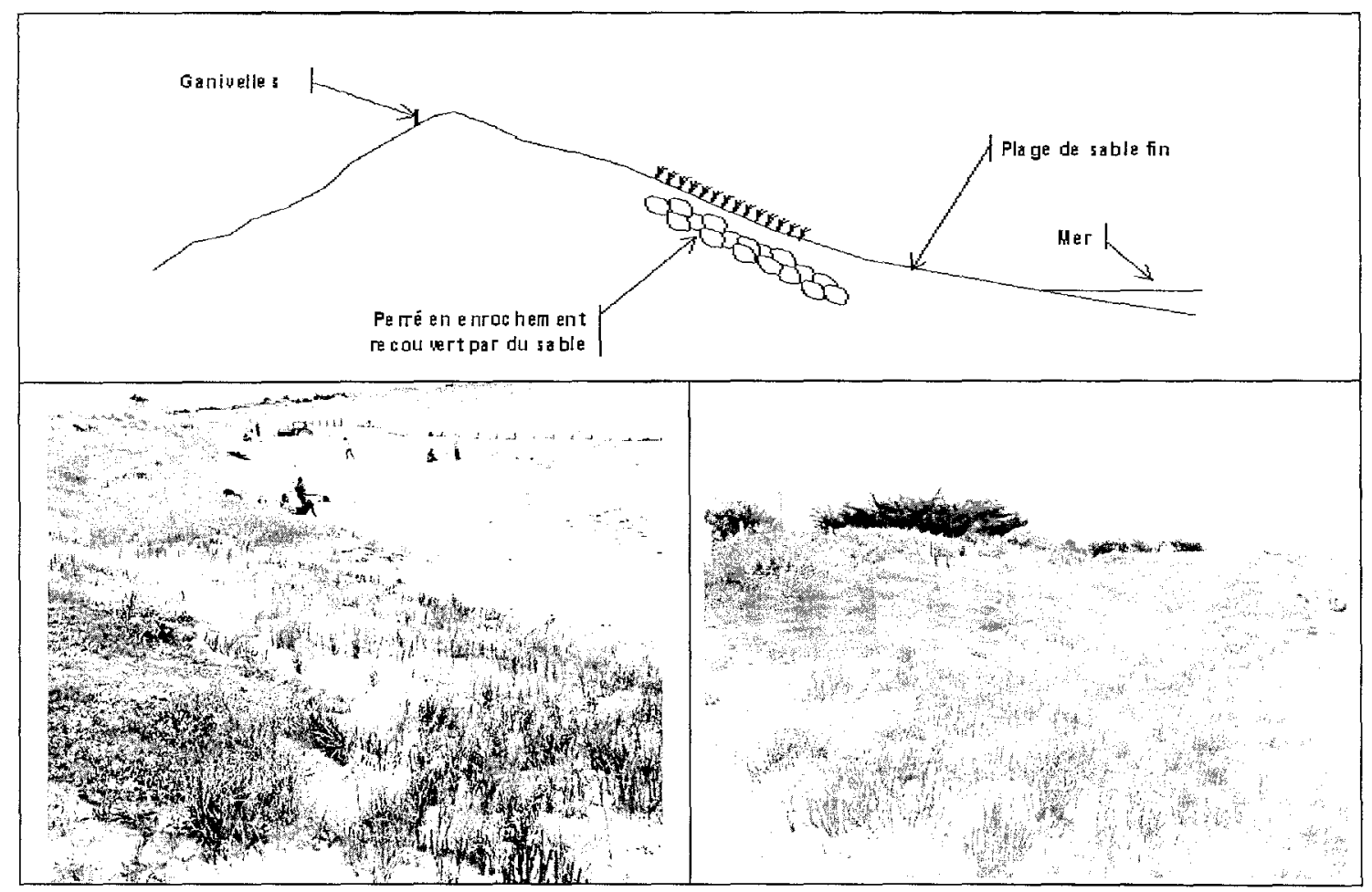

Figure 3. Schéma typologique : Plage des Sables d'Or

L'ensemble du littoral a été survolé en hélicoptère pour réaliser un reportage photographique et un film. Ces prises de vues ont été très utiles pour cartographier les zones peu accessibles, et aussi lors des réunions de travail et de présentation des études, en illustration des commentaires des bureaux d'études.

\subsection{Classes typologiques}

Les ouvrages ont été classés en 10 classes typologiques définissant aussi bien des ouvrages artificiels (digues, talus, épis, murs) que des ouvrages "naturels" (falaise, dunes), représentés sur les fiches typologiques et les plans d'ensemble par des couleurs. Des pictogrammes ont permis de mieux définir des sous-classes typologiques précisant la constitution des "revêtements" (pierres pavées, enrochements, béton, maçonneries), des crêtes d'ouvrages (muret).

\section{Etude de diagnostic des ouvrages}

Une nouvelle inspection visuelle des ouvrages a été réalisée sur les zones les plus exposées et sur les ouvrages les plus sensibles. Le diagnostic porte sur un linéaire défini par une typologie d'ouvrage et une zone exposée à des conditions hydrodynamiques homogènes. Chaque ouvrage présentant des désordres, ou ne répondant pas aux contraintes des nouvelles données hydrodynamiques (liées à l'augmentation des niveaux d'eau extrêmes de projet), a fait l'objet d'une fiche pathologique(figure 4) basée sur des schémas et un reportage photo. 


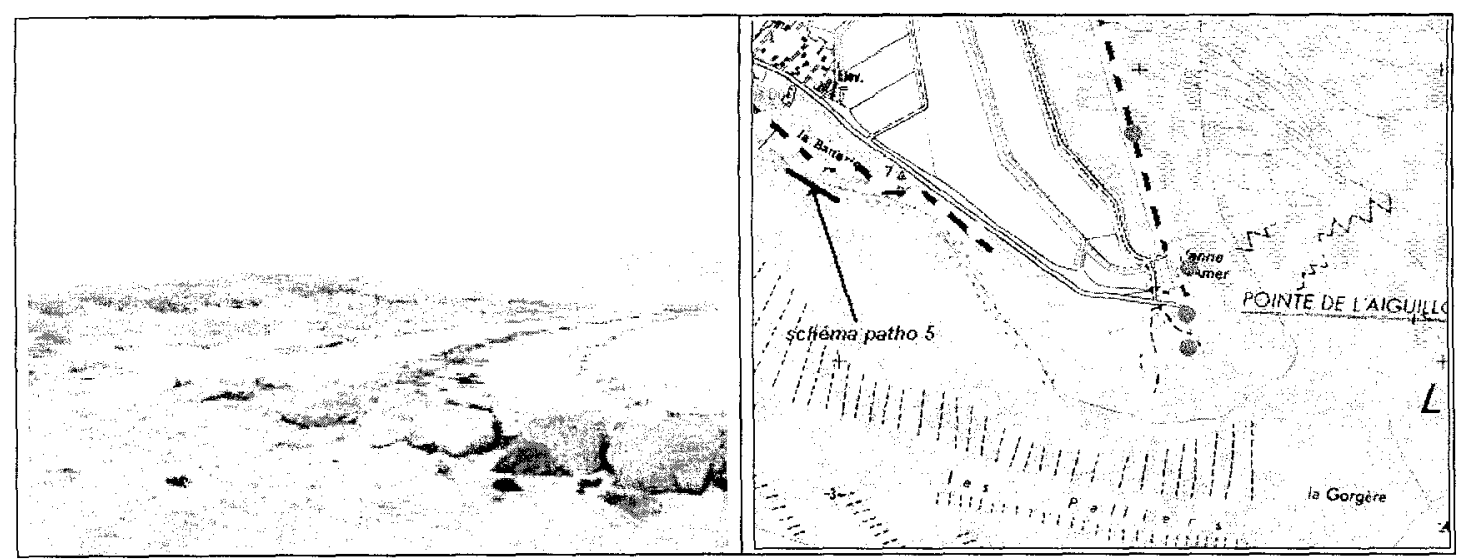

Figure 4. Fiche pathologique - Pointe de l'Aiguillon

Les ouvrages ont été classés par familles de pathologie pour proposer par classe pathologique les principes de restauration et définir les urgences.

\begin{tabular}{|c|c|c|c|}
\hline Classe 0 & blanc & Absence de Pathologie & \\
\hline Classe 1 & rouge & Altimétrie insuffisante & $\begin{array}{l}\text { Débordement, affouillement en arrière de } \\
\text { l'ouvrage, érosion, destruction de la } \\
\text { végétalisation }\end{array}$ \\
\hline Classe 2 & jaune & $\begin{array}{l}\text { Faiblesse de la structure } \\
\text { externe de l'ouvrage }\end{array}$ & $\begin{array}{l}\text { Erosion, désolidarisation, déplacement, } \\
\text { départ d'éléments de la structure, } \\
\text { basculement du muret, infiltration d'eau }\end{array}$ \\
\hline Classe 3 & violet & $\begin{array}{c}\text { Faiblesse de la structure } \\
\text { interne de l'ouvrage }\end{array}$ & $\begin{array}{l}\text { Tassements, glissements, fissures, } \\
\text { circulation d'eau }\end{array}$ \\
\hline Classe 4 & marron & Faiblesse du sous-sol & $\begin{array}{c}\text { Tassements, affaissements, circulation } \\
\text { d'eau, }\end{array}$ \\
\hline Classe 5 & bleu & $\begin{array}{l}\text { Faiblesse liée aux actions } \\
\text { hydrodynamiques }\end{array}$ & $\begin{array}{l}\text { Fissurations, basculement du muret, } \\
\text { érosion, départ d'éléments de la structure } \\
\text { externe de l'ouvrage }\end{array}$ \\
\hline Classe 6 & vert & $\begin{array}{l}\text { Faiblesse liée aux actions } \\
\text { externes (ni climatiques, ni } \\
\text { météorologiques) }\end{array}$ & $\begin{array}{l}\text { Trous, ravines, ornières, piétinement, } \\
\text { départ de la végétalisation }\end{array}$ \\
\hline
\end{tabular}

\section{Principes de restauration et schéma de définition des urgences}

\subsection{Principes de restauration}

Les principes de restauration sont donnés en fonction des pathologies et de la typologie, ainsi que des contraintes liées aux actions hydrodynamiques. Une ou deux solutions de restauration ont été proposées par typologie et pathologie.

Pour les digues des polders, les principales idées directrices de restauration sont de rehausser ces ouvrages et de créer un chemin d'entretien sur la face coté large, ce chemin permet de renforcer la structure de l'ouvrage et limite les franchissements (niveau d'eau et exposition aux houles). Les digues des étiers sont aussi rehaussées pour prendre en compte les nouvelles consignes de niveau d'eau 
extrême. Pour les zones naturelles à restaurer, il est systématiquement proposé des méthodes douces: ré-ensablement de la plage et des dunes, protection des dunes, mise en place de perrés bas enfouis sous la dune (dernier rempart), si leur mise en cuvre est possible.

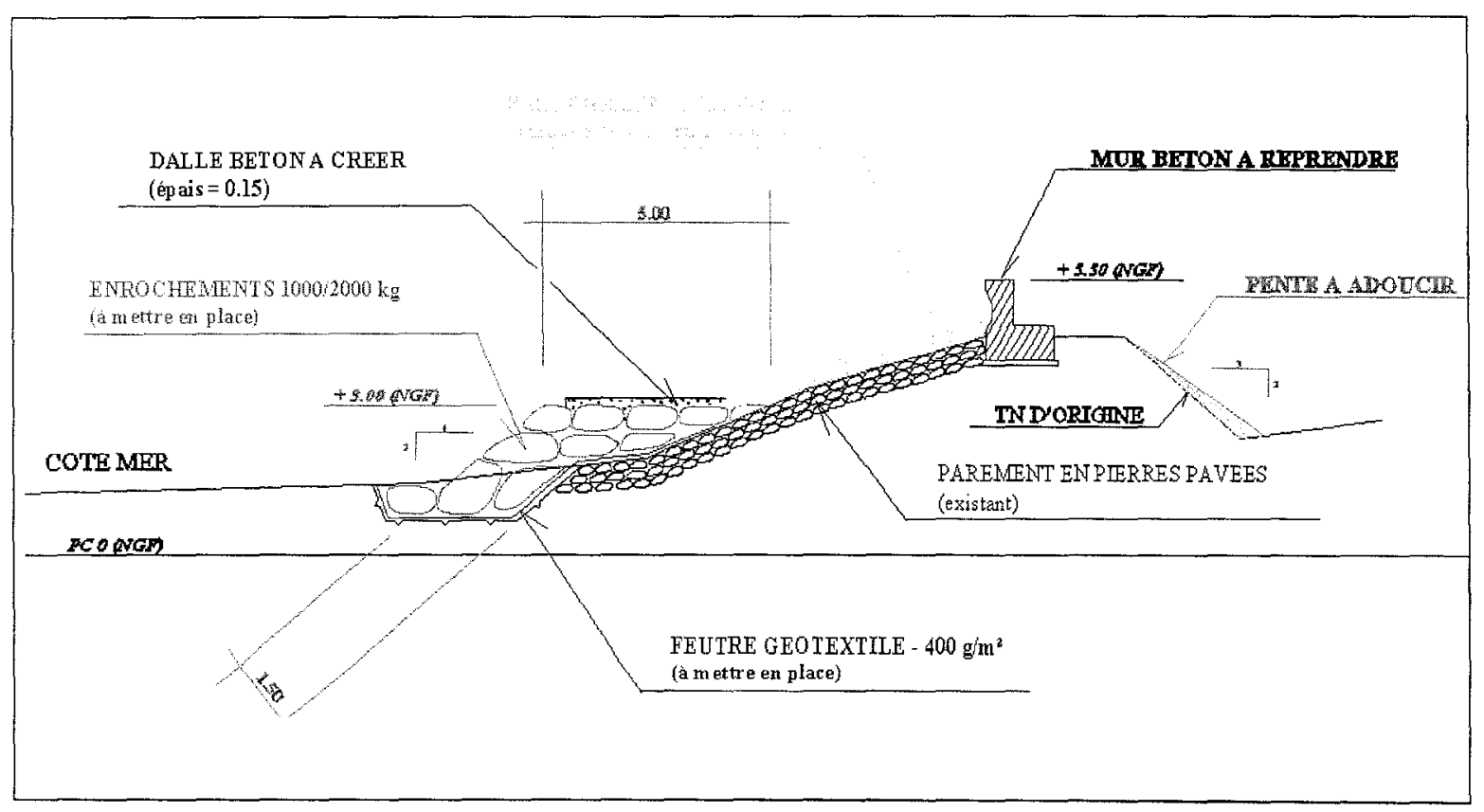

Figure 4. Coupe type de restauration d'une digue de polder

\subsection{Définition des urgences}

Les urgences des travaux ont été hiérarchisées en fonction de la vulnérabilité de la zone protégée par l'ouvrage, et du degré de service de l'ouvrage. Cette approche est similaire à celle d'un Plan de Prévention des Risque du littoral, mais simplifiée. Elle a pour simple but de définir les travaux urgents à programmer dans les prochaines années (10 millions d'Euros). Il a été fait une distinction entre les ouvrages naturels (dunes et falaises) et les ouvrages artificiels.

L'exemple ci-après est propre aux ouvrages artificiels qui protègent les zones poldérisées de la baie de Bourgneuf.

La notion de vulnérabilité des zones à protéger est liée aux facteurs de l'occupation des sols et de l'activité. Trois classes ont été utilisées: faible, moyenne et forte. Le degré de service de l'ouvrage est un indicateur qui permet de voir comment l'ouvrage assure ses fonctions initiales. Il est approché par le diagnostic. Six classes de service de l'ouvrage ont été définis, notés de 1 à 6 , (1 ouvrage assurant sa fonction; 6 ouvrage n'assurant plus sa fonction). 
La grille ci dessous permet de définir en fonction du degré de service de l'ouvrage et de la vulnérabilité les urgences classées de 1 à 5

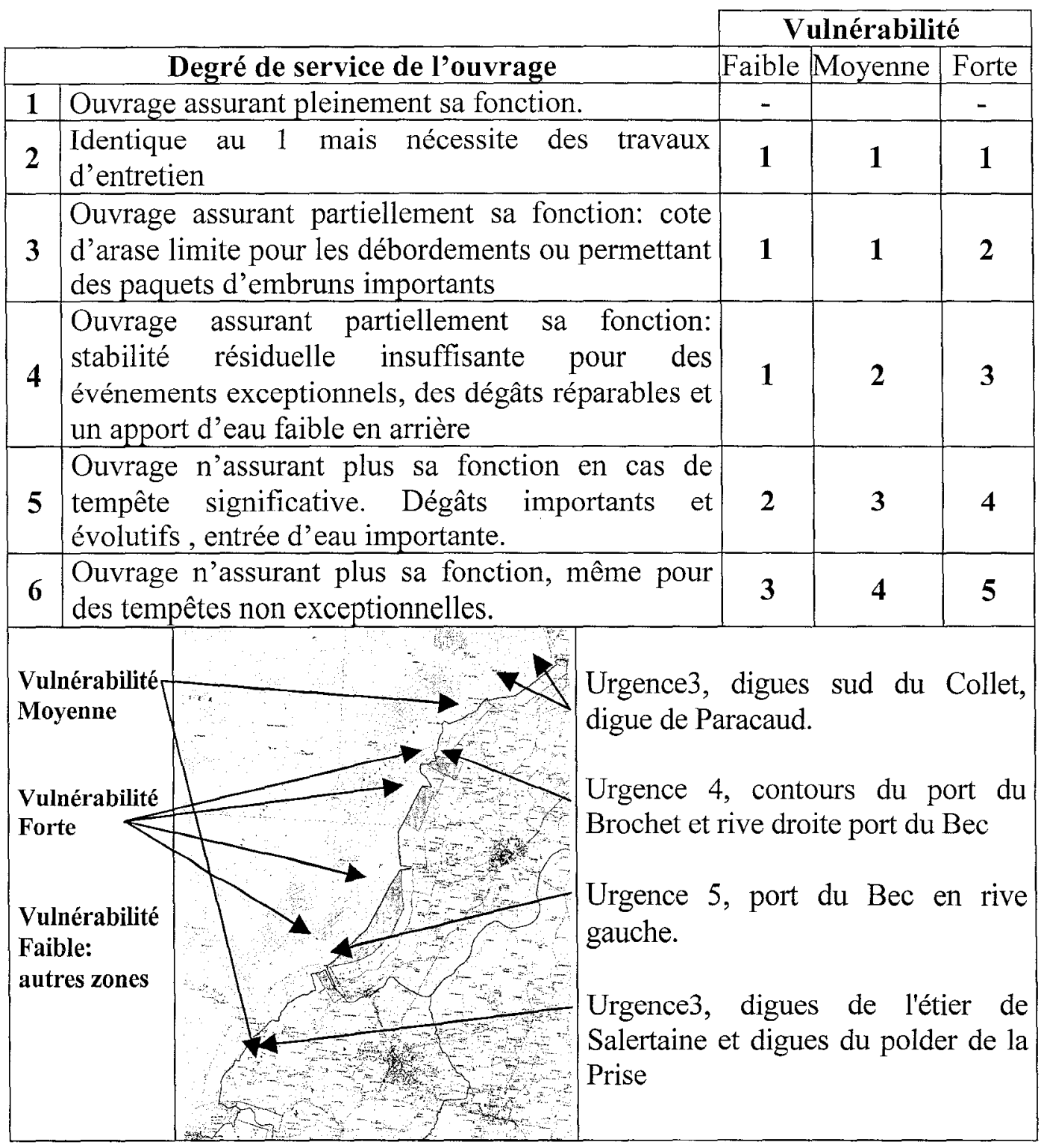

\section{Méthodologie de maintien des connaissances du patrimoine littoral}

La réalisation du diagnostic des ouvrages de défense contre la mer du littoral Vendéen a comporté la collecte, la mise en forme et la conservation de divers types de données. Les données de localisation au format SIG permettent l'édition de cartes thématiques; les données graphiques documentaires permettent l'édition de fiches de synthèse. Nous avons utilisé le logiciel MapInfo pour les données de localisation. Les données graphiques sont sous forme de fichiers DXF/DWG (dessin), JPEG/BMP (images), DOC/XLS, PPT (présentation). L'arborescence des données et les noms des fichiers sont organisés pour faciliter 
leur repérage. Ainsi, les noms des fichiers contiennent entre autres le numéro INSEE de la commune. Cette organisation a été mise en place pour faciliter l'intégration de ces données dans le SIG du Conseil Général de Vendée.

Le maintien des connaissances est aussi basé sur une réactualisation des données intégrant des visites de terrains pour prendre en compte l'évolution des ouvrages et de leur environnement. Ainsi, il est programmé des visites de contrôle dans le but de déterminer si une intervention de maintenance est nécessaire ou non.

$\checkmark$ La visite annuelle consiste en une inspection visuelle de l'ouvrage. Elle se déroulera à basse mer après la période des tempêtes hivernales. Les ouvrages seront comparés à l'état de référence (état zéro). Les désordres seront répertoriés dans les fiches de référence, photographies à l'appui. Son coût est estimé à 1000 Euros par km de rivage.

$\checkmark$ La visite quinquennale comprend des contrôles sur les zones ayant fait l'objet de remarques suite aux visites annuelles: vérifications de l'altimétrie de l'ouvrage (tassements), état des sous-couches (renard, migration de matériaux), état des parements (béton, enrochements), etc... Un relevé cartographique des secteurs dunaires et de plages permettra de suivre l'évolution du trait de rivage.

Une visite sera réalisée après chaque tempête exceptionnelle sur les zones exposées. Elle permettra d'évaluer les dégâts avec la possibilité d'engager des réparations immédiates. Des moyens de locomotion rapides devront donc être prévus à cet effet (vélo, moto, hélicoptère...).

\section{Références}

[1] "Statistiques des niveaux marins extrêmes le long des côtes de France" SHOM: rapport 001/94.

[2] "Evolution du niveau moyen de la mer et fréquence des tempêtes: certitudes et incertitudes". Communication au séminaire SHF du 05-06-01 P.A. Pirazzoli

[3] SHOM. Etude spécifique de niveaux marins extrêmes - étude sur l'élévation du niveau des mers rapport BCEOM.

[4] IPCC (Intergovernmental Panel on Climate Change) : organisme international chargé de suivre l'évolution du climat. Site: www.ipcc.ch 